\title{
Surprising Relations between Parametric Level Correlations and Fidelity Decay
}

\author{
H. Kohler, ${ }^{1}$ I. E. Smolyarenko, ${ }^{2}$ C. Pineda,${ }^{3}$ T. Guhr, ${ }^{1}$ F. Leyvraz,${ }^{3}$ and T. H. Seligman ${ }^{3}$ \\ ${ }^{1}$ Fachbereich Physik, Universität Duisburg-Essen, D-47057 Duisburg, Germany \\ ${ }^{2}$ School of Information Systems, Computing and Mathematics, Brunel University, Uxbridge UB8 3PH, United Kingdom \\ ${ }^{3}$ Instituto de Ciencias Físicas, Universidad Nacional Autónoma de México, Cuernavaca, Mexico \\ (Received 20 December 2007; revised manuscript received 20 February 2008; published 14 May 2008)
}

Relations among fidelity, cross-form-factor (i.e., parametric level correlations), and level velocity correlations are found both by deriving a Ward identity in a two-matrix model and by comparing exact results, using supersymmetry techniques, in the framework of random matrix theory. A power law decay near Heisenberg time, as a function of the relevant parameter, is shown to be at the root of revivals recently discovered for fidelity decay. For cross-form-factors the revivals are illustrated by a numerical study of a multiply kicked Ising spin chain.

PACS numbers: 03.65.Sq, 03.65.Yz, 05.30.Ch, 05.45.Mt

Fidelity decay presently attracts considerable attention [1]. It measures the change of quantum dynamics of a state under a modification of the Hamiltonian. In quantum information, fidelity measures the deviation between a mathematical algorithm and its physical implementation. From a different point of view, important insight into the properties of the underlying systems is provided by the studies of correlations between spectra of random and/or chaotic Hamiltonians which differ by a parameterdependent perturbation [2]. Since statistical properties of fidelity decay in random or chaotic systems involve both spectra and eigenfunctions of the original and perturbed Hamiltonians, the existence of any connections between fidelity and purely spectral correlations is not a priori obvious.

Random matrix theory (RMT) has been successful in describing quantum many-body systems and as a model for the spectral properties of single particle systems whose classical analogue is chaotic [3]. Within RMT fidelity was analyzed in linear response approximation [4] and both fidelity [5-7] and parametric correlations [8] were calculated exactly using the supersymmetry method. An unexpected fidelity revival at Heisenberg time was encountered [5] within RMT and confirmed in a dynamical coupled spin chain model [9].

Earlier, differential relations between parametric spectral correlations and parametric density correlations were established $[10,11]$. By relating the latter to the fidelity amplitude via Fourier transform, we show in this Letter that the existence of these relations opens a crucial insight into the properties of fidelity decay. By analyzing the characteristic features of the parametric correlations in the time domain, the cross-form-factor, we discover a new, simple interpretation of the previously puzzling phenomenon of revival [5]. These relations follow directly from the basic definitions and symmetries of the underlying matrix models, being essentially Ward identities. We show that they are valid under very general assumptions. No explicit (e.g., supersymmetric) calculation is required; however, they rely on the universality of the parametric spectral correlations at the scale of mean level spacing. We thus explain the origin of various relations connecting spectral and wave-function correlations, and establish a unified framework for their analysis and generalizations. A relation between fidelity decay and level velocity correlation function is given. The latter is important from the experimental point of view, being used for independent access to system parameters. We confirm the general results comparing fidelity decay and cross-form-factors in RMT. We illustrate our analytical results with a numerical study of a multiply kicked Ising spin chain.

We consider Hamiltonians modeled by $N \times N$ matrices

$$
H^{ \pm}(\lambda)=H \pm \lambda V / 2,
$$

where $H$ and $V$ are independently drawn from ensembles of the same symmetry. In particular, $V$ is drawn from the Gaussian orthogonal ensemble (GOE), the Gaussian unitary ensemble (GUE), or the Gaussian symplectic ensemble (GSE) ensembles of RMT, labeled $\beta=1,2,4$. The ensemble average over both is indicated by angular brackets. It is convenient to fix the variances as $\left\langle H_{i j} H_{k l}\right\rangle=$ $D^{-1}\left\langle V_{i j} V_{k l}\right\rangle$ where $D$ is the mean level spacing of $H^{ \pm}(0)$ in the energy region of interest. In the RMT case, $D=\pi^{2} / N$ in the center of the spectrum. The mean level spacing is then $\lambda$ independent up to corrections of order $1 / N$. By construction, $H^{ \pm}(\lambda)$ is in the same symmetry class as $H$ for any $\lambda$.

The parametric two-level correlation function is defined as

$$
\tilde{R}_{\beta}\left(E^{+}, E^{-}, \lambda\right)=\sum_{n, m}\left\langle\delta\left(E^{-}-\epsilon_{n}^{-}(\lambda)\right) \delta\left(E^{+}-\epsilon_{m}^{+}(\lambda)\right)\right\rangle .
$$

It is mapped onto a dimensionless energy scale, where the mean level spacing is rescaled to unity. One has

$$
\tilde{X}_{\beta}(r, \lambda)=\lim _{N \rightarrow \infty} D^{2} \tilde{R}_{\beta}\left(E^{+}, E^{-}, \lambda\right),
$$


which solely depends on the difference $r=\left(E^{+}-E^{-}\right) / D$. The cross-form-factor is obtained as a Fourier transform

$$
\tilde{K}_{\beta}(t, \lambda)=\int_{-\infty}^{+\infty}\left[1-\tilde{X}_{\beta}(r, \lambda)\right] e^{2 \pi t t r} d r, \quad t>0 .
$$

Time $t$ is measured in units of Heisenberg time $t_{H}=D^{-1}$. Fidelity decay is expressed via the echo operator [1]

$$
M(t, \lambda)=\exp \left[\imath 2 \pi t H^{-}(\lambda) / D\right] \exp \left[-\imath 2 \pi t H^{+}(\lambda) / D\right] .
$$

Its expectation value with a given state is the fidelity amplitude and its average

$$
f_{\beta}(t, \lambda)=\frac{1}{N} \operatorname{tr}\langle M(t, \lambda)\rangle
$$

is a measure for the difference in the two time evolutions as a function of $\lambda$.

The functions in Eq. (3) were calculated exactly with the supersymmetry method [8] for $\beta=1,2,4$. The Fourier transforms are (see also [12])

$$
\begin{aligned}
\tilde{K}_{1}(t, \lambda)= & \int_{\max (0, t-1)}^{t} d u \int_{0}^{u} d v \frac{2 t^{2}(t-u)(1-t+u)}{\left(v^{2}-t^{2}\right)^{2}} \\
& \times \frac{\exp \left(-2 \pi^{2} \lambda^{2}\left[2 u t+t-t^{2}+v^{2}\right]\right)}{\sqrt{\left(u^{2}-v^{2}\right)\left(u^{2}+2 u+1-v^{2}\right)}}, \\
\tilde{K}_{2}(t, \lambda)= & \frac{\exp \left(-2 \pi^{2} \lambda^{2} t^{1+\theta(t-1)}\right)}{2 \pi^{2} \lambda^{2} t} \sinh \left(2 \pi^{2} \lambda^{2} t^{2-\theta(t-1)}\right), \\
\tilde{K}_{4}(t, \lambda)= & t^{2} \int_{-1}^{+1} d u \int_{0}^{1-|u|} d v \frac{(u+t)^{2}-1}{\left(t^{2}-v^{2}\right)^{2}} \\
& \times \frac{v \theta(u-1+t) \exp \left(-\pi^{2} \lambda^{2}\left[t^{2}-v^{2}+2 t u\right]\right)}{\sqrt{\left[(u-1)^{2}-v^{2}\right]\left[(u+1)^{2}-v^{2}\right]}},
\end{aligned}
$$

with Heaviside's $\theta$ function. For $\lambda=0$ the cross-formfactors reduce to the standard form factors $K_{\beta}(t)$ [3], i.e., $\tilde{K}_{\beta}(t, 0)=K_{\beta}(t)$. In Fig. 1 we show $\tilde{K}_{\beta}(t, \lambda)$ versus time $t$ for two values of $\lambda$. For $\lambda=0.1$, the correlations vanish as $t \rightarrow \infty$. A second peak develops in the GSE case for $\lambda=1$ at $t=2$. The singularity at $t=1$ persists. For the GOE and for the GUE cases finite peaks appear at $t=1$ but not at multiples thereof. For all ensembles another peak appears for small times $t \ll 1$. Its location scales asymptotically with $\lambda^{-2}$.

The peak appearing at $t=1$ for large $\lambda$ clearly indicates that here the correlations decay more slowly as a function of $\lambda$ than at all other times $t$. We study this in more detail by an asymptotic analysis in $\lambda$ of the exact integral expressions (7). We calculate the weight $W_{\beta}(t, \lambda)=$ $\lambda^{2} \int_{t-\lambda^{-2}}^{t+\lambda^{-2}} \tilde{K}_{\beta}\left(t^{\prime}, \lambda\right) d t^{\prime}$ of the peaks at $t=1$ and, for the GSE, also at $t=2$. In contrast to the peak height the weight is well defined for all times for all three ensembles. We find
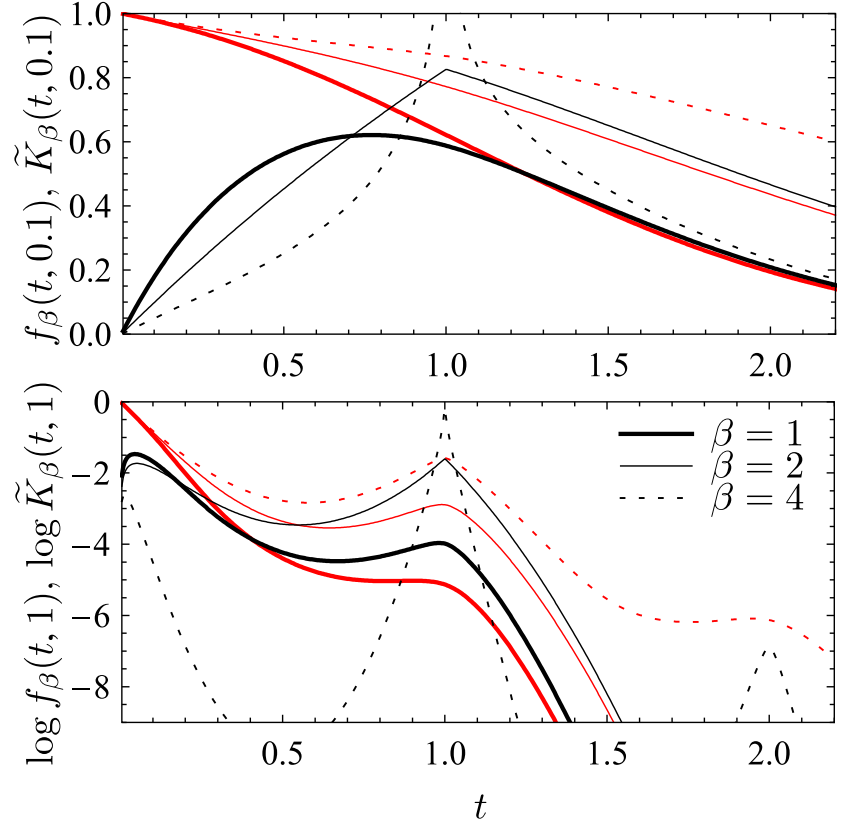

FIG. 1 (color online). Cross-form-factor (black) and fidelity [gray (red)] versus time for two different values $\lambda$. The results for the three Gaussian ensembles $\beta=1,2,4$ are given as thick solid, thin solid, and dashed lines, respectively.

$$
W_{\beta}(1, \lambda) \propto \lambda^{-2(4-\beta) / \beta}+\mathcal{O}\left(\lambda^{-8 / \beta}\right)
$$

and $W_{4}(2, \lambda) \propto \lambda^{-6}$. The weight of the first peak $t \approx 0$ scales as $\lambda^{-2}$ independently of the ensemble. These decays are governed by power laws in $\lambda$ while they are exponential for all other times. We shall see below that the behavior of the cross-form-factor at $t=1$ is directly related to fidelity revivals, which for the GSE also occur at $t=2$.

For the classical ensembles $K_{\beta}(t)$ is nonanalytic at $t=1$ [3]. The degree $\mathcal{D}(g, x)$ of nonanalyticity of a function $g(x)$ at $x$ is defined as the smallest integer $\mathcal{D}$ for which the $\mathcal{D}$ th derivative $g^{(\mathcal{D})}$ is discontinuous at $x$. For the form factor we find $\mathcal{D}\left(K_{4}, 1\right)=0, \mathcal{D}\left(K_{2}, 1\right)=1$, and $\mathcal{D}\left(K_{1}, 1\right)=$ $\mathcal{D}\left(K_{4}, 2\right)=3$. For typical times we find $\mathcal{D}\left(K_{\beta}, t\right)=\infty$, because $K_{\beta}(t)$ is analytic. We thus arrive at a relation between the asymptotic behavior of $W_{\beta}(t, \lambda)$ for large perturbation to the degree of nonanalyticity of $K_{\beta}(t)$ which reads

$$
W_{\beta}(t, \lambda) \propto \lambda^{-2 \mathcal{D}\left(K_{\beta}, t\right)}+\cdots, \quad t>0 .
$$

We conjecture that this relation also holds for arbitrary $\beta \neq 1,2,4$.

We use the multiply kicked Ising (MKI) spin chain proposed in $[9,13]$ to illustrate the revival in the crossform-factor. The MKI spin chain is a periodic 1D array of $L$ spins $1 / 2$ with antiferromagnetic nearest-neighbor Ising interaction of unit strength and periodic boundary conditions. Each spin receives periodically two different kicks of instantaneous magnetic field pulses. The time-reversal 
breaking Floquet operator of the system is $U_{\mathrm{MKI}}=$ $U_{\mathrm{I}} U_{\mathrm{K}}^{(1)} U_{\mathrm{I}} U_{\mathrm{K}}^{(2)}$, where $U_{\mathrm{I}}$ is the time evolution operator of the unkicked spin chain and $U_{\mathrm{K}}^{(n)}=\exp \left(-\imath \sum_{j} \vec{b}^{(n)} \cdot \vec{\sigma}_{j}\right)$ $(n=1,2)$ describes each magnetic pulse with a dimensionless magnetic field $\vec{b}^{(n)}$. Here $\vec{\sigma}_{j}$ are the Pauli operators for particle $j$. The translational symmetry $\left(\vec{\sigma}_{j} \rightarrow \vec{\sigma}_{j+1}\right)$ foliates the space in different symmetry sectors. For the choice $\vec{b}^{(1)}=(0,1,1)$ and $\vec{b}^{(2)}=(1.4,0,1.4)$ the spectral statistics in most symmetry sectors display excellent agreement with the GUE. We introduce an additional magnetic pulse of strength $\delta$ in $z$ direction as a perturbation. We define $U_{\delta}=U_{\mathrm{MKI}} \exp \left(\iota \delta \sum_{j=0}^{L-1} \sigma_{j}^{z}\right)$ and calculate the cross-form-factor of $U_{\mathrm{MKI}}$ and $U_{\delta}$ using direct diagonalization, omitting the problematic sectors. The perturbation strength $\lambda$ can be calculated from $\delta$ using the correlation functions of the perturbing operator [1]. Details are given elsewhere.

In Fig. 2 we compare results of this model with RMT results of Eq. (7). We see good agreement with the theoretical result, up to statistical fluctuations, measured by the imaginary part. In particular the peak at $t=1$ is observed.

We now derive the announced differential relations connecting the cross-form-factor with fidelity, deferring the reader to a follow-up paper for more details. Consider a general 4-point parametric correlation function $F_{\alpha \beta ; \gamma \delta}\left(z_{1}, z_{2}\right)=\left\langle\mathcal{F}_{\alpha \beta ; \gamma \delta}\left(z_{1}, z_{2} ; H^{-}, H^{+}\right)\right\rangle$defined as

$$
F_{\alpha \beta ; \gamma \delta}\left(z_{1}, z_{2}\right)=\left\langle\left(\frac{1}{z_{1}-H^{-}}\right)_{\alpha \beta}\left(\frac{1}{z_{2}-H^{+}}\right)_{\gamma \delta}\right\rangle .
$$

The definition of the angular brackets is now expanded to denote either the average over an arbitrary matrix ensemble with measure $d \nu(H)$ or the energy averaging over a spectral window of an individual quantum chaotic system. We

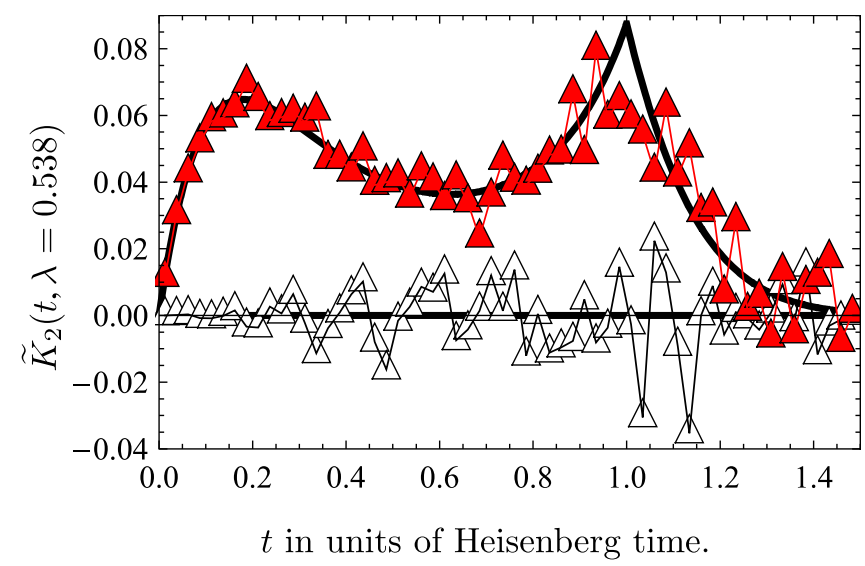

FIG. 2 (color online). The cross correlation function for the MKI model with $L=18$. Filled (empty) triangles correspond to the real (imaginary) part of the cross correlation. The statistical error (measured by the imaginary part) is small enough to observe clearly the peak. The theoretical expectation Eq. (7) is plotted as a thick curve. do not require $d \nu(H)$ to be Gaussian or even rotationally invariant. The distribution of $V$, on the other hand, is required to be Gaussian in order to ensure the existence of the announced differential relations at finite order [see Eq. (16) below]. The Fourier transform of fidelity amplitude corresponds to $F_{\alpha \beta ; \beta \alpha}$ and parametric spectral correlator corresponds to $F_{\alpha \alpha ; \beta \beta}$, with a summation over double indices. Introducing $H_{1,2}$ via $\delta\left(H_{1,2}-H^{ \pm}(\lambda)\right)$, Fourier transforming the matrix $\delta$ functions and integrating over $V$, the averages are rewritten as

$$
\begin{aligned}
\langle\mathcal{F}\rangle= & \int d \Lambda_{1} d \Lambda_{2} d \nu(H) d H_{1} d H_{2} \\
& \times e^{\mathrm{tr}\left[\imath \Lambda_{1}\left(H_{1}-H\right)+\imath \Lambda_{2}\left(H_{2}-H\right)-\left(\lambda^{2} D^{2} / 4 \beta\right)\left(\Lambda_{2}-\Lambda_{1}\right)^{2}\right]} \mathcal{F},
\end{aligned}
$$

where the symmetry class of the matrices $\Lambda_{1}, \Lambda_{2}$ corresponds to the symmetry class of $H$, and multiple factors of $2 \pi$ are absorbed into the definition of $d \Lambda_{1,2}$.

The invariance of the flat integration measures $d H_{1,2}$ with respect to independent shifts in $H_{1}$ and $H_{2}$ implies

$$
\left\langle\operatorname{tr}\left(\frac{\partial}{\partial H_{1}}-\frac{\partial}{\partial H_{2}}\right)^{2} \mathcal{F}_{\alpha \alpha ; \beta \beta}\right\rangle=-\left\langle\operatorname{tr}\left(\Lambda_{1}-\Lambda_{2}\right)^{2} \mathcal{F}_{\alpha \alpha ; \beta \beta}\right\rangle
$$

The full measure in Eq. (11) is also approximately invariant under a simultaneous shift of $H_{1}$ and $H_{2}$. The violation of this symmetry stems from the noninvariance of $d \nu(H)$ under the shifts of $H$. However, universality implies that the correlation functions depend on such shifts only through the average density of states and level velocity variance [8]. This dependence is thus manifested only on time scales much shorter than $t_{H}$, which is of interest here. In invariant unitary RMT ensembles universality under shifts was shown in [14]. Although not yet proved in general, no violations of this universality are known. In particular, universality follows automatically in models which allow for field theoretical representations of correlation functions [15]. With these caveats we can set

$$
\left\langle\operatorname{tr}\left(\frac{\partial}{\partial H_{1}}+\frac{\partial}{\partial H_{2}}\right)^{2} \mathcal{F}_{\alpha \alpha ; \beta \beta}\right\rangle \approx 0
$$

and combine with Eq. (12) to

$$
4\left\langle\operatorname{tr} \frac{\partial}{\partial H_{1}} \frac{\partial}{\partial H_{2}} \mathcal{F}_{\alpha \alpha ; \beta \beta}\right\rangle=\left\langle\operatorname{tr}\left(\Lambda_{1}-\Lambda_{2}\right)^{2} \mathcal{F}_{\alpha \alpha ; \beta \beta}\right\rangle .
$$

Using

$$
\begin{gathered}
\left\langle\operatorname{tr} \frac{\partial}{\partial H_{1}} \frac{\partial}{\partial H_{2}} \mathcal{F}_{\alpha \alpha ; \beta \beta}\right\rangle=\frac{\partial^{2}}{\partial z_{1} \partial z_{2}} F_{\alpha \beta ; \beta \alpha}, \\
\left\langle\operatorname{tr}\left(\Lambda_{1}-\Lambda_{2}\right)^{2} \mathcal{F}_{\alpha \alpha ; \beta \beta}\right\rangle=-\frac{4 \beta}{D^{2}} \frac{\partial}{\partial \lambda^{2}} F_{\alpha \alpha ; \beta \beta}
\end{gathered}
$$

and Fourier transforming $F$, we finally show that 


$$
\frac{\partial}{\partial \lambda^{2}} \tilde{K}_{\beta}(t, \lambda)=-\frac{4 \pi^{2} t^{2}}{\beta} f_{\beta}(t, \lambda),
$$

under very general assumptions. The averaged fidelity amplitude has been calculated in Ref. [5] for the GOE and the GUE. For the GSE,

$$
\begin{aligned}
f_{4}(t, \lambda)= & \int_{-1}^{+1} d u \int_{0}^{1-|u|} d v \frac{(u+t)^{2}-1}{\left(t^{2}-v^{2}\right)^{2}}|v| \theta(u-1+t) \\
& \times \frac{\left(t^{2}-v^{2}+2 t u\right) \exp \left(-\pi^{2} \lambda^{2}\left[t^{2}-v^{2}+2 t u\right]\right)}{\sqrt{\left[(u-1)^{2}-v^{2}\right]\left[(u+1)^{2}-v^{2}\right]}} .
\end{aligned}
$$

A direct comparison of the exact expressions for $\tilde{K}_{\beta}(t, \lambda)$ obtained in the present contribution and of the ones for $f_{\beta}(t, \lambda)$ in Ref. [5] and in Eq. (18) confirms the validity of Eq. (17) in the universal RMT regime (although we stress that it is valid for any disordered or chaotic model which exhibits a separation of scales between the oscillatory local and smooth global behavior of spectral statistics).

Relation (17) allows us to view fidelity revival at Heisenberg time $t_{H}$ as being rooted in the algebraic decay of the cross-form-factor. Furthermore, due to the established relations, power law decay as a function of $\lambda$ must also hold for fidelity at $t_{H}$ and, for the GSE, at $2 t_{H}$. This could also have been derived directly from the exact equations.

In Fig. 1, we show the fidelity amplitude. Similar to the behavior of the cross-form-factor, a peak at $t=1$ appears for all three ensembles [5], and for increasing $\beta$ the peaks become more and more pronounced. In the GSE case, a second peak emerges at $t=2$. This peak was not seen in the numerics of [5] as it was beyond numerical accuracy.

Relation (17) is, essentially, a Ward identity associated with the action (11). It immediately allows one to establish a connection between fidelity amplitude and the Fourier transform of the level velocity correlator $C(t, \lambda)$, which is related to $\tilde{K}$ by a Ward identity $-4 \pi^{2} t^{2} C(t, \lambda)=$ $\left(\partial^{2} / \partial \lambda^{2}\right) \tilde{K}(t, \lambda)$ (see, e.g., [16]). As seen from Eq. (11), $\tilde{K}$ is a function of $\lambda^{2}$; it follows from (17) after a short calculation that

$$
\beta C(t, \lambda)=\left(2+4 \lambda^{2} \frac{\partial}{\partial \lambda^{2}}\right) f(t, \lambda) .
$$

To summarize, we established relations between crossform-factor and level velocities on the one hand and fidelity decay on the other hand. They hold in any system displaying universality of spectral correlations. The present formalism can be used to construct a whole family of Ward identities relating apparently unconnected correlation functions. One instance is generalizations of the "optical theorem" found in [11], which relates fidelity amplitude for small perturbations to the spectral form factor $K_{\beta}(t)$. Further, the results presented here do not apply to crossover regimes, where $V$ changes the symmetry of $H$. One such relation was obtained using supersymmetry methods in [10]. A broader set of differential relations, generalizing those of [10], can be obtained by utilizing different transformation properties of the action Eq. (11) under symmetry-preserving and symmetry-violating shifts. Details of these and other hierarchies of relations will be presented elsewhere.

Our findings make it possible to explain features of one quantity via the other, i.e., the characteristics of fidelity decay in terms of the cross-form-factor or vice versa. In particular, the revivals of both quantities are linked in this way. We studied in detail the decay laws of the corresponding peaks. Further peaks are not possible. The very occurrence of the peaks in the cross-form-factors is neither trivial nor intuitive and will be discussed in elsewhere.

We thank T. Gorin, J. Keating, T. Prosen, D. Savin, R. Schäfer, and H.J. Stöckmann for useful discussions. We acknowledge Grants DFG-KO3538/1-1 (H. K.), DFGSFB Transregio 12 (T. G., H. K.), EPSRC-EP/E037429/1 (I. S.), PAPIIT IN112507, and CONACyT 57334 (T.S., C.P.).

[1] T. Gorin et al., Phys. Rep. 435, 33 (2006).

[2] B. D. Simons et al., Phys. Rev. Lett. 72, 64 (1994); B. D. Simons et al., Phys. Rev. Lett. 71, 2899 (1993); M. Faas et al., Phys. Rev. B 48, 5439 (1993); B. Dietz et al., Phys. Lett. A 215, 181 (1996).

[3] T. Guhr et al., Phys. Rep. 299, 189 (1998).

[4] T. Gorin et al., New J. Phys. 6, 20 (2004).

[5] H.-J. Stöckmann and R. Schäfer, New J. Phys. 6, 199 (2004); Phys. Rev. Lett. 94, 244101 (2005).

[6] T. Gorin et al., Phys. Rev. Lett. 96, 244105 (2006).

[7] H. J. Stöckmann and H. Kohler, Phys. Rev. E 73, 066212 (2006).

[8] B. D. Simons and B.L. Altshuler, Phys. Rev. Lett. 70, 4063 (1993); Phys. Rev. B 48, 5422 (1993); B. D. Simons et al., Phys. Rev. B 48, 11450 (1993).

[9] C. Pineda et al., Phys. Rev. E 73, 066120 (2006).

[10] N. Taniguchi et al., Europhys. Lett. 29, 515 (1995); N. Taniguchi et al., Phys. Rev. B 53, R7618 (1996).

[11] N. Taniguchi et al., Phys. Rev. Lett. 75, 3724 (1995).

[12] E. R. Mucciolo et al., Phys. Rev. B 49, 15197 (1994).

[13] T. Prosen, Phys. Rev. E 65, 036208 (2002).

[14] I. E. Smolyarenko and B. D. Simons, J. Phys. A 36, 3551 (2003).

[15] K. B. Efetov, Supersymmetry in Disorder and Chaos (Cambridge University Press, Cambridge, England, 1996), 1st ed.

[16] J. T. Chalker et al., J. Math. Phys. (N.Y.) 37, 5061 (1996). 